$\begin{array}{lllllllllllll}\text { A C T A } & \text { C H E M I C A S C A N D I N A V I C A } 12 & (1958) & 983-994\end{array}$

\title{
Determination of Specific Gravity with Divers and Hydrometers with Loose Stem
}

\author{
S Ø R E N B R G and J. J. V. L U N D B E R G
}

The Laboratory of Porcelcensfabriken "Danmark" Ltd., Lyngby-Copenhagen, Denmark

\begin{abstract}
A new method has been developed for determining the specific gravity of liquids, with divers or floats which may be loaded with metal weights to attain the floating equilibrium. The diver may also be provided with a loose stem of metal wire so that the instrument may function as a hydrometer.

Generally, only two divers are required for the measurements, viz. one of density 0.75 and another of density 0.95. Both divers are so dimensioned that their weights and volumes approximately satisfy an equation indicated in the article. Under these conditions, the specific gravity of the liquid may be calculated with sufficient accuracy from the results of the measurements by the diver method as well as from the results of measurements by the hydrometer method, using only a slide rule.

The random error of the diver method is about $0.9 \times 10^{-5}$. The random error of the hydrometer method is greater, and further a systematic error is introduced, e. $g$. because of adhesion between the fluid and the stem. The order of magnitude of this systematic error may be determined by comparative measurements according to the diver method and the hydrometer method.
\end{abstract}

$\mathrm{M}$ any investigators have tried to measure the specific gravity of fluids by applying bodies of known specific gravity, so-called divers, which during the measurements are completely submerged. The floating equilibrium of the diver is attained by addition of platinum weights to the diver, by alteration of the temperature of the fluid, by diluting the fluid, etc.

Divers have particularly been used for determining the specific gravity of sea water ${ }^{1-4}$ and heavy water etc. ${ }^{5-11}$ and in the fineness analysis ${ }^{12-17}$ to determine the specific gravity in sedimenting suspensions, where the application of divers is advantageous for two reasons. First, in a heterodisperse, sedimenting suspension the specific gravity increases from the surface downwards, and there will therefore always be a level at which a diver with lower specific gravity than the homogeneous suspension, but with higher specific gravity than the suspending medium, may attain the floating equlibrium. Such special arrangements to attain the floating equilibrium may therefore be omitted, when a series of divers of different specific gravities are available. 
Second, a consideration of the sedimentation process shows that a diver, which is in equilibrium in a sedimenting suspension, will move downwards with the same velocity as the largest particles present at the level of its geometric centre of gravity. I.e. it moves downwards faster than the sedimenting velocity of every particle present above this level. As a hydrometer, therefore, it will not be overtaken by sedimenting particles and systematic errors caused by deposition of particles onto the diver will be very small.

Finally, it may be mentioned that Nansen ${ }^{3}$ has proposed to provide the diver with a loose stem, which, when the diver is in equilibrium, protrudes through the surface of the fluid so that the instrument is altered to a hydrometer (with loose stem).

With the exception of the examination of the specific gravity in sedimenting suspensions by the fineness analysis, it seems as if the method is not widely used for the determination of the specific gravity of fluids in spite of its very great precision and the quickness of the measurements. This is probably due to the fact that hitherto it has been necessary to calcralate the specific gravity from a non-logarithmic expression by the application of logarithms with at least five figures.

It may, however, be shown that by applying two divers, the weights and volumes of which both satisfy a later indicated equation, one may calculate the specific gravity of the fluid with sufficient accuracy without other calculating facilities than a slide rule. By this method the specific gravity is determined with a random error of $\pm 0.9 \times 10^{-5}$ and the method is therefore more precise than the hydrometer method and Westphal's balance. It is further possible to make the method still more accurate, but this will require a very precise thermostat and the application of more than two divers, if the calculations are still to be simply made.

By providing the diver with a loose stem of platinum wire, for instance, one can, with moderate decrease in preciseness, make the measurements according to the hydrometer method. To obtain a satisfactory preciseness in this case, it is necessary to carry out comparative measurements according to the diver method and the hydrometer method for each new fluid which may be examined in order to determine the systematic error of the hydrometer method and to be able to correct for it. These errors are particularly due to adhesion between the fluid and the stem, to changes in the condition of the surface of the stem, and to fluid attached to the protruding part of the stem.

In the following:

$a$ indicates the weight of the diver (in vacuo),

$\varrho_{20,20}$ the specific gravity of the unloaded diver at $20^{\circ} \mathrm{C}$

$\varrho_{20,4}$ compared to water at $20^{\circ}$ and $4^{\circ} \mathrm{C}$, respectively,

$\alpha_{20,20}$ the increase in specific gravity of the diver at $20^{\circ} \mathrm{C}$

$\alpha_{20,4}$ compared to water at $20^{\circ} \mathrm{C}$ and $4^{\circ} \mathrm{C}$, due to the load,

$p_{20,20}$ the specific gravity of the load at $20^{\circ} \mathrm{C}$ compared

$p_{20,4}$ to water at $20^{\circ} \mathrm{C}$ and $4^{\circ} \mathrm{C}$, respectively,

$z_{20,20}$ an arbitrary specific gravity at $20^{\circ} \mathrm{C}$ compared

$z_{20,4}$ to water at $20^{\circ} \mathrm{C}$ and $4^{\circ} \mathrm{C}$, respectively. 
When a diver is in floating equilibrium in a medium with the load $x$ one has:

and since

$$
\frac{a+x}{\frac{a}{\varrho_{20,4}}+\frac{x}{p_{20,4}}}=\varrho_{20,4}+\alpha_{20,4}
$$

one gets

$$
z_{20,4}=z_{20,20} \times 0.99823
$$

$$
\frac{a+x}{\frac{a}{\varrho_{20,20}}+\frac{x}{p_{20,20}}}=\varrho_{20,20}+\alpha_{20,20}
$$

from which may be deduced

and

$$
\frac{a+x}{\frac{a p_{20,20}}{\varrho_{20,20}}+x}=\frac{\varrho_{20,20}+\alpha_{20,20}}{p_{20,20}}
$$

and

$$
\begin{aligned}
& \frac{a+x}{a\left(\frac{p_{20,20}}{\varrho_{20,20}}-1\right)}=\frac{\varrho_{20,20}+\alpha_{20,20}}{p_{20,20}-\varrho_{20,20}-\alpha_{20,20}}=\frac{1+\frac{\alpha_{20,20}}{\varrho_{20,20}}}{\frac{p_{20,20}}{\varrho_{20,20}}-\frac{{ }^{\prime 20,20}}{\varrho_{20,20}}-1} \\
& \text { Multiplying by }\left(\frac{p_{20,20}}{\varrho_{20,20}}-1\right) \text { one gets } \\
& \frac{a+x}{a}=\frac{\frac{p_{20,20}}{\varrho_{20,20}}+\frac{p_{20,20} \cdot \alpha_{20,20}}{\varrho_{20,20}}-\frac{\alpha_{20,20}}{\varrho_{20,20}}-1}{\frac{p_{20,20}}{\varrho_{20,20}}-\frac{\alpha_{20,20}}{\varrho_{20,20}}-1}
\end{aligned}
$$

from which may be derived

and we have

$$
1+\frac{x}{a}=1+\frac{\frac{p_{20,20} \cdot \alpha_{20,20}}{\varrho_{20,20}}}{\frac{p_{20,20}}{\varrho_{20,20}}-\frac{\alpha_{20,20}}{\varrho_{20,20}}-1}
$$

$$
x=\frac{a \cdot \alpha_{20,20}}{\frac{\varrho_{20,20}^{2}}{p_{20,20}}\left(\frac{p_{20,20}}{\varrho_{20,20}}-\frac{\alpha_{20,20}}{\varrho_{20,20}}-1\right)}
$$

$$
x=\frac{a \cdot \alpha_{20,20}}{\varrho_{20,20}\left(1-\frac{\varrho_{20,20}-\alpha_{20,20}}{p_{20,20}}\right)}
$$

Acta Chem. Scand. 12 (1958) No. 5 
from which may be derived

$$
\alpha_{20,20}=\frac{x \cdot \varrho_{20,20}\left(1-\frac{\varrho_{20,20}}{p_{20,20}}\right)}{a\left(1+\frac{\varrho_{20,20} \cdot x}{a \cdot p_{20,20}}\right)}
$$

(3) is the non-logarithmic expression by which the difference between the specific gravity of the loaded and the unloaded diver may be calculated.

$x$ being the load which corresponds to the floating equilibrium in water at $20^{\circ} \mathrm{C}$, we have

$$
\varrho_{20,20}+\alpha_{20,20}=1
$$

By substituting (4) into (2) it may be shown that

$$
\alpha_{20,20}=\frac{1}{1+\frac{a \cdot p_{20,20}}{x\left(p_{20,20}-1\right)}}
$$

Using (4) and (5), the specific gravity of the unloaded diver may be determined by floating experiments in water since only divers with lower specific gravity than water are used.

Eqn. (3) may be written

$$
\alpha_{20,20}=\frac{x \cdot \varrho_{20,20}\left(p_{20,20}-\varrho_{20,20}\right)}{a \cdot p_{20,20}\left(1+\frac{\varrho_{20,20} \cdot\left(p_{20,20}-\varrho_{20,20}\right) \cdot x}{a \cdot p_{20,20}\left(p_{20,20}-\varrho_{20,20}\right)}\right)}
$$

Simplifying, we introduce

which gives

$$
\frac{a \cdot p_{20,20}}{\varrho_{20,20}\left(p_{20,20}-\varrho_{20,20}\right)}=C
$$

As

$$
\alpha_{20,20}=\frac{x}{C} \frac{1}{1+\frac{x}{C\left(p_{20,20}-\varrho_{20,20}\right)}}
$$

$$
\frac{1}{1+\beta}=1-\frac{\beta}{1+\beta}
$$

where $\beta$ is an arbitrary number, eqn. (7) may be written:

and

$$
\alpha_{20,20}=\frac{x}{C}\left(1-\frac{\frac{x}{C\left(p_{20,20}-\varrho_{20,20}\right)}}{1+\frac{x}{C\left(p_{20,20}-\varrho_{20,20}\right)}}\right)
$$

$$
\alpha_{20,20}=\frac{x}{C}\left(1-\frac{1}{1+\frac{C}{x}\left(p_{20,20}-\varrho_{20,20}\right)}\right)
$$


and we further have:

$$
\alpha_{20,20}=\frac{x}{C}-\frac{\left(\frac{x}{C}\right)^{2}}{\frac{x}{C}+p_{20,20}-\varrho_{20,20}}
$$

When the weight and specific gravity of the diver are so chosen that $C$ is a whole number, for instance 10,20 or $50, x / C$ may conveniently be calculated by mental calculation and as the second term is smaller than 0.00350 except in special cases, it may be calculated with sufficient preciseness by a slide rule.

It may further be shown that when

$\frac{x}{C}<0.1$ and $p_{20,20}-\varrho_{20,20}>20$ or $\frac{x}{C}<0.08$ and $p_{20,20}-\varrho_{20,20}>15$ the following approximate equation may be used

$$
\alpha_{20,20}=\frac{x}{C}-\frac{\left(\frac{x}{C}\right)^{2}}{p_{20,20}-\varrho_{20,20}}
$$

When $C$ is not exactly a whole number

$$
C=N(1+\varepsilon)
$$

where $\varepsilon$ is a small number and $N$ is a whole number.

When $\varepsilon<0.001 x / C$ may be computed by mental calculation by the following approximate equation

$$
\frac{x}{C}=\frac{x(1-\varepsilon)}{N}
$$

The diver is made preferably from thermometer glass (Jena 16 III) to minimize the thermal after-effect, or from quartz, and the upper tip of the diver is drawn out into a short glass stem. The instrument is shown on Fig. 1 without and with the metal stem. Q is a ballast of mercury. $\mathrm{M}$ is a horizontal plate of soft iron, supported by a rod $S$ fixed by shellac to the inside of the diver's lower tip. The diver may therefore be manipulated from the outside with a magnet when it is in floating equilibrium.

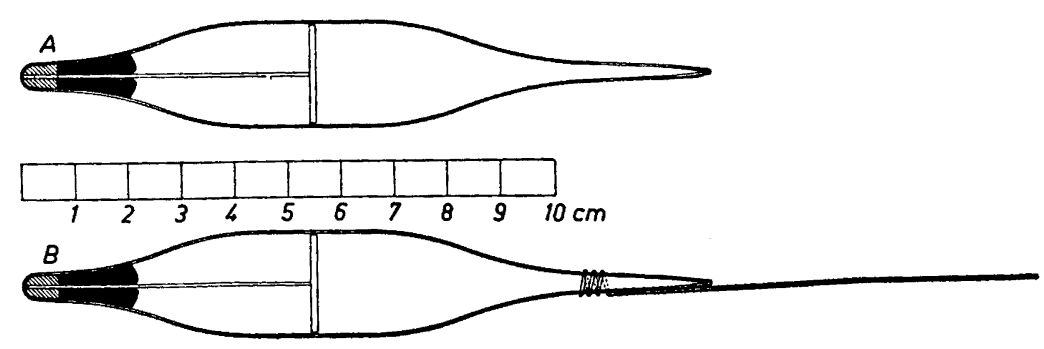

Fig. 1. A: Diver. B: Hydrometer with loose stem.

Acta Chem. Scand. 12 (1958) No. 5 
To secure that $C$ and $\varrho_{20,20}$ do not differ more than $1 \%$ from $N$ and the desired specific gravities, 0.75 and 0.95 , respectively, the following course of procedure may be applied: From (6) we have

$$
\frac{a}{\varrho_{20,20}}=C \frac{p_{20,20}-\varrho_{20,20}}{p_{20,20}}=Y
$$

where $Y$ is the weight of the water displaced by the diver at $20^{\circ} \mathrm{C}$. As

$$
\varrho_{20,20}=\frac{\varrho_{20,4}}{0.99823}
$$

where 0.99823 is the density of water at $20^{\circ} \mathrm{C}$, we further have

$$
\frac{a}{0.99823 \varrho_{20,20}}=\frac{a}{\varrho_{20,4}}=\frac{C\left(p_{20,20}-\varrho_{20,20}\right)}{0.99823 p_{20,20}}=V
$$

where $V$ is the volume of the diver. From (11) and (12) it is seen, that $Y$ and $V$ are determined when $C$ and $\varrho_{20,20}$ are chosen.

Before the stem of the diver is cut off to the desired length, the diver is put down in a graduated glass filled to a mark with mercury and the approximate volume of the diver is read from the rising of the mercury. The stem is then cut off to such a length that the volume of the diver is somewhat greater than the volume calculated by (11). Enough mercury is now introduced into the diver so that the latter may float submerged in water at $20^{\circ} \mathrm{C}$ with the stem closed by a small rubber hood. The stem is then sealed with a flame and by loading the diver with weights of platinum it is brought to floating equilibrium in water at $20^{\circ} \mathrm{C}$.

From the load and the weight of the diver its specific gravity and volume may be calculated by (3), (4) and (12). By etching with hydrofluoric acid and by new floating experiments, the volume of the diver may now be decreased to approximately the calculated volume.

The tip of the stem is then cut off and sufficient mercury is removed from the diver to bring its specific gravity approximately to the desired one. The stem is now once more sealed in a flame. By new floating experiments and repeated etching, it is now possible to adjust both the $C$-value and the specific gravity of the diver to the desired values within a tolerance of less than $1 \%$. This is sufficient, but it is of course necessary afterwards to determine both values with a tolerance of less than about $0.5 \times 10^{-5}$ by loading experiments in water by $20^{\circ} \mathrm{C}$. These determinations may conveniently be repeated up to 10 times in order to give a good average.

For the measurements, one diver with a specific gravity of about 0.75 and another with a specific gravity of about 0.95 are applied and $\alpha_{20,20}$ is computed by (8) or (9) and (10).

For the most part, ringshaped weights of platinum are used which may be conveniently placed around the glass stem of the diver. Weights smaller than $10 \mathrm{mg}$ should be made from stainless steel or titanium, preferably, (densities 7.85 and 4.5, respectively) provided that these materials are not corroded by the liquid to be investigated. The reason is that such small platinum weights (density 21.5), $5 \mathrm{mg}$ and less, are rather difficult to handle. 
As stainless steel and titanium have almost the same appearance as platinum, it is also advantageous to make 10,20 , and $50 \mathrm{mg}$ weights of gold (density 19.3).

The weights of stainless steel, titanium and gold are made sufficiently more heavy than the corresponding weights of platinum so that the buoyancy of the weights in water is the same. All these weights being small, their relative accuracy may be rather limited, and thus in many cases it is not necessary to correct for the difference in buoyancy when the medium has a specific gravity different from 1 .

For weights of stainless steel of specific gravity 7.85 and weight $R$ the correction $\Delta R$ is calculated from the following formula, when the specific gravity of the medium is $\sigma_{20,20}$ :

$$
\Delta R=0.1\left(1-\sigma_{20,20}\right) R
$$

For weights of gold of specific gravity 19.3 and weight $G$ the corresponding formula is

$$
\Delta G=0.005\left(1-\sigma_{20,20}\right) G
$$

Big weights may be made of tantalum (density 16.6) instead of platinum as tantalum is very wear-resistant and almost as resistant against corrosion as gold and platinum.

To estimate loads smaller than the smallest weights applied, one may interpolate from the ascending and sinking velocities of the diver near the floating equilibrium. The velocity varies approximately directly with the load.

The medium which is to be examined is filled into a glass container which is placed in a water thermostat regulated at $20^{\circ} \mathrm{C}$. Before the determinations, the diver with load must be freed from air bubbles by brushing it with a brush with soft hairs. To avoid a greater randomerror than $\pm 0.5 \times 10^{-5}$ the thermostat must regulate with a tolerance of $\pm 0.02^{\circ} \mathrm{C}$.

In the examination of mixtures it is necessary to take precautions against alteration of the composition by evaporation of one of the components.

To save time it is advantageous to provide the diver with a loose stem, ordinarily of platinum wire, with a winding at one of its ends, so that it may be fixed around the glass stem of the diver. In computing the load which the stem represents, it must be taken into consideration that the buoyancy is acting only on that part of the stem which is situated under the surface of the fluid. Since the volume of that part of the stem which is above the surface of the fluid is ordinarily small, it is very often possible to estimate the weight of the corresponding volume of fluid with sufficient accuracy without knowing the accurate density of the fluid. When this weight is added to the weight of the stem, we get the weight of loads submerged in the fluid which corresponds to the actual weight of the stem, provided the stem and the loads are of the same material. The calculation of $\alpha_{20,20}$ may then be carried out as by the diver method.

Before the stem is placed on the diver, the latter must be loaded with such a weight that it will come into floating equilibrium when part of the stem is above the surface of the fluid. With some practice this may be estimated by observing how large a part of the glass stem is protruding from the surface of the fluid before the platinum stem is placed on the diver.

Acta Chem. Scand. 12 (1958) No. 5 
The surface tension of water being about $70 \mathrm{dyn} / \mathrm{cm}$, the influence of the surface tension of a fluid of the same surface tension as water on a stem of diameter $0.5 \mathrm{~mm}$ may under certain conditions amount to

$$
0.05 \pi \cdot 70=11 \mathrm{dyn}(c a .11 \mathrm{mg})
$$

It is therefore necessary to determine the systematic error of the hydrometer method for every new fluid by comparative measurements with the apparatus according to the diver method and the hydrometer method.

\section{EXPERIMENTAL}

The diver method. To determine the specific gravity of the divers used and the random error of the diver method, the load was determined by floating equilibrium in water at $20 \pm 0.02^{\circ} \mathrm{C}$, ten times with a diver of density about 0.75 and ten times with a diver of density about 0.95 .

From the average loads of the two divers their specific gravities are calculated from (4) and (5) and their $C$-velues from (6).

To determine the random error of the diver method the specific gravity of the water examined has been calculated from (8) and (4) as well as the deviations $\lambda$ of the single measurements from the average (1.000000). These deviations are numerically the same as the deviations from the average one would find when calculating from (8) and (4) as well as the specific gravity of the diver $\varrho_{20,20}$, the increase in specific gravity of the diver $a_{\mathbf{2 0}, \mathbf{2 0}}$ corresponding to the single measurement. From the $\lambda$-values it is therefore possible to calculate the random error $\mu \mathrm{d}, \mathrm{m}$ of the specific gravity of the diver (taken as an average of ten measurements)

$$
\mu_{\mathrm{d}, \mathrm{m}}=\sqrt{\frac{\sum_{1 \lambda^{2}}^{\mathrm{n}}}{\mathrm{n}(\mathrm{n}-1)}}
$$

in which $\mathrm{n}$ is 10 .

Further it is possible to calculate the random error $\mu$ of a single determination of the specific gravity of the water from the formula

in which $\mathrm{n}=10$

$$
\mu=\sqrt{\frac{\sum_{1}^{n} \lambda_{2}}{n(n-1)}+\frac{\sum_{1}^{n} \lambda_{2}}{n-1}}=\sqrt{\frac{(n+1) \sum_{1}^{n} \lambda_{2}}{n(n-1)}}
$$

The results of the experiments are given in Tables 1 and 2.

The hydrometer method. To determine the systematic as well as the random error of the hydrometer method the specific gravity of distilled water at $20 \pm 0.02^{\circ} \mathrm{C}$ has been determined according to the hydrometer method with the two divers, the specific gravity of which were determined by ten floating experiments according to the diver method.

Three platinum stems with the diameters $0.5,0.3$ and $0.2 \mathrm{~mm}$ were used and ten experiments were carried out with each stem in combination with each diver. The total load on the diver was calculated as the weight in $\mathrm{g}$ of the load + the weight in $\mathrm{g}$ of the stem + the volume of the protruding stem in $\mathrm{cm}^{3}$. From the total load, the specific gravity of the water is calculated from (8) and (4) disregarding the systematic error of the hydrometer method.

The quantity $K$ defined by the equation

$$
K=1-\varrho_{20,20}-a_{20,20}
$$


Table 1. Diver method, diver No. 8, weight of diver in vacuo $14.4679 \mathrm{~g}$.

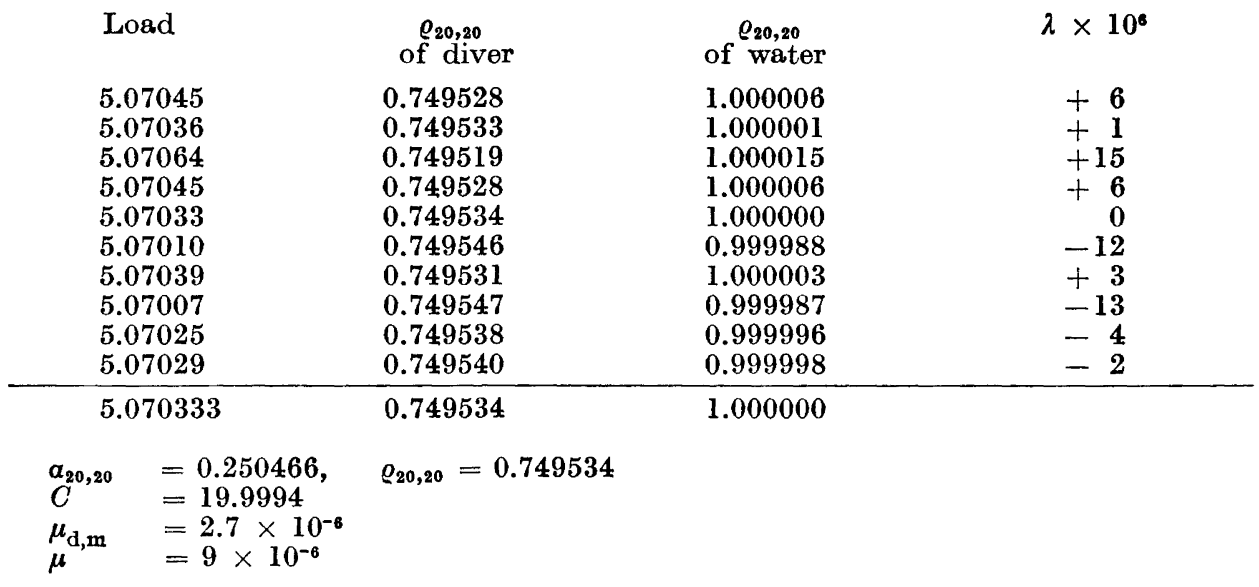

Table 2. Diver method, diver No. 37, weight of diver in vacuo $18.1646 \mathrm{~g}$.

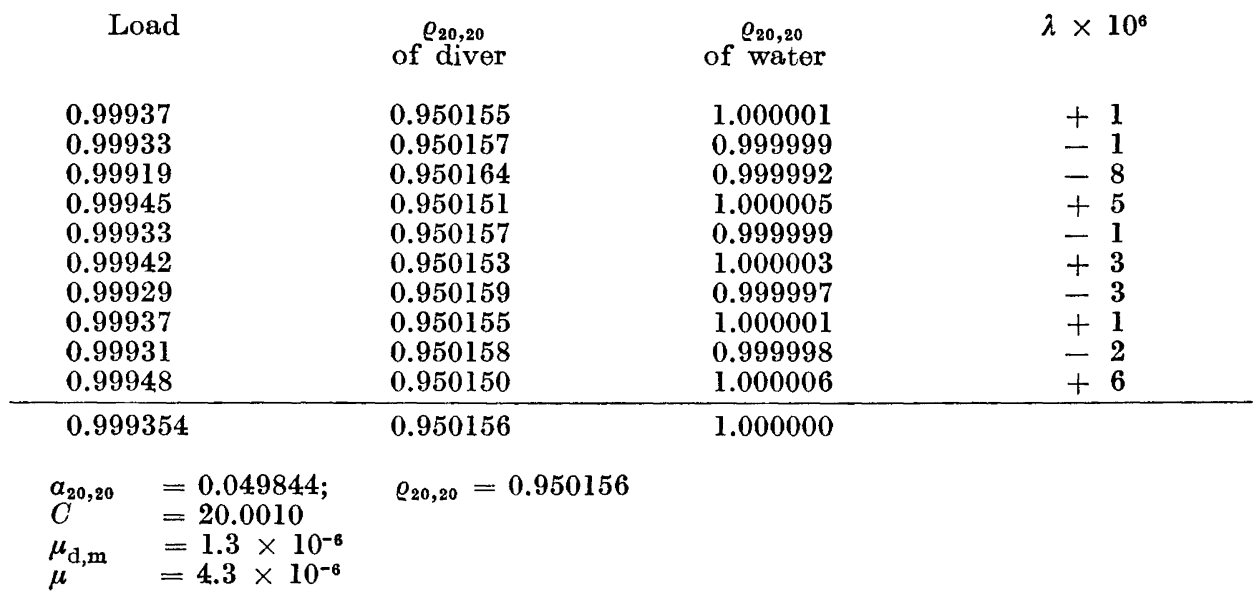

is then tabulated and the average $K_{\mathrm{m}}$ of the $K$-values is calculated.

A corrected value $\varrho_{f, 20,20}$ of the specific gravity of the water is determined from the equation

$$
\varrho_{\mathrm{f}, 20,20}=\varrho_{\mathrm{m}, 20,20}+K_{\mathrm{m}}+\alpha_{20,20}
$$

Here $\varrho_{\mathrm{m}, 20,20}$ is the specific gravity of the diver determined by the diver method as an average of 10 determinations.

The differences $\lambda$ between the corrected specific gravities of the water and 1.000000 are tabulated.

These differences are numerically the same as the differences one would get by subtracting $K_{\mathrm{m}}$ from the individual $K$-values and by subtracting the average of the $a_{20,20^{-}}$ values from the individual $a_{\mathbf{2 0 , 2 0}}$-values.

The random error $\mu \mathrm{K}_{\mathrm{m}}$ of the quantity $K_{\mathrm{m}}$, which is approximately the systematic error of the hydrometer method, may therefore be calculated from the formula

Acta Chem. Scand. 12 (1958) No. 5 
Table 3. Hydrometer method, diver No. 8 , diameter of stem $0.5 \mathrm{~mm}, \varrho_{20,20}=0.749534$, $C=19.9994$; weight of stem $0.500 \mathrm{~g}$.

\begin{tabular}{|c|c|c|c|c|c|c|}
\hline $\begin{array}{l}\text { Load } \\
\mathrm{g}\end{array}$ & $\begin{array}{c}\text { Protruding } \\
\mathrm{cm}^{3} \\
\times 10^{4}\end{array}$ & $\begin{array}{l}\text { Total } \\
\mathrm{g}\end{array}$ & $\begin{array}{c}\varrho_{20,20} \text { with } \\
\text { syst.error }\end{array}$ & $K \times 10^{5}$ & $\begin{array}{c}\varrho_{20,20} \text { corrected } \\
\text { for } \\
\text { syst.error }\end{array}$ & $\lambda \times 10^{5}$ \\
\hline 4.564 & 32 & 5.0672 & 0.99985 & 15 & 0.99997 & -3 \\
\hline 4.563 & 30 & 5.0660 & 0.99979 & 21 & 0.99991 & -9 \\
\hline 4.563 & 66 & 5.0696 & 0.99997 & 3 & 1.00009 & +9 \\
\hline 4.564 & 30 & 5.0670 & 0.99984 & 16 & 0.99996 & -4 \\
\hline 4.563 & 60 & 5.0690 & 0.99994 & 6 & 1.00006 & +6 \\
\hline 4.562 & 64 & 5.0684 & 0.99991 & 9 & 1.00003 & +3 \\
\hline 4.564 & 32 & 5.0672 & 0.99985 & 15 & 0.99997 & -3 \\
\hline 4.565 & 44 & 5.0694 & 0.99996 & 4 & 1.00008 & +8 \\
\hline 4.563 & 34 & 5.0664 & 0.99981 & 19 & 0.99993 & -7 \\
\hline 4.563 & 46 & 5.0676 & 0.99987 & 13 & 0.99999 & -1 \\
\hline$f, 20.20$ & $\begin{array}{l}\times 10^{-6} \\
6 \times 10^{-6}\end{array}$ & $\begin{array}{r}50.6778 \\
5.06778\end{array}$ & 0.999876 & $\begin{array}{r}121 \\
12\end{array}$ & & \\
\hline
\end{tabular}

Table 4. Hydrometer method, diver No. 8, diameter of stem $0.3 \mathrm{~mm}$, $\varrho_{20,20}=0.749532, C=19.9994$; weight of stem $0.200 \mathrm{~g}$.

\begin{tabular}{rccrrrr} 
Load & $\begin{array}{c}\text { Protruding } \\
\mathrm{cm}^{3} \\
\times 10^{4}\end{array}$ & Total & $\begin{array}{c}\varrho_{20,20} \text { with } \\
\text { syst.error }\end{array}$ & $K \times 10^{5} \varrho_{20,20} \begin{array}{c}\text { corrected } \\
\text { for } \\
\text { syst.error }\end{array}$ & $\begin{array}{c}\text { sor } \\
4.864\end{array}$ \\
49 & 5.0689 & 0.99993 & 7 & 1.00004 & +4 \\
4.864 & 37 & 5.0677 & 0.99987 & 13 & 0.99998 & -2 \\
4.864 & 31 & 5.0671 & 0.99984 & 16 & 0.99995 & -5 \\
4.865 & 35 & 5.0685 & 0.99991 & 9 & 1.00002 & +2 \\
4.864 & 32 & 5.0672 & 0.99985 & 15 & 0.99996 & -4 \\
4.864 & 50 & 5.0690 & 0.99994 & 6 & 1.00005 & +5 \\
4.865 & 29 & 5.0679 & 0.99988 & 12 & 0.99999 & -1 \\
4.865 & 31 & 5.0681 & 0.99989 & 11 & 1.00000 & 0 \\
4.865 & 38 & 5.0688 & 0.99993 & 7 & 1.00004 & +4 \\
4.864 & 34 & 5.0674 & 0.99986 & 14 & 0.99997 & -3 \\
\hline
\end{tabular}

$\mu_{\varrho_{\mathrm{f}, 20,20}}=38 \times 10^{-6}$

Table 5. Hydrometer method, diver No. 8, diameter of stem $0.2 \mathrm{~mm}$, $\varrho_{20,20}=0.749534, C=19.9994$; weight of stem $0.100 \mathrm{~g}$.

\begin{tabular}{ccccccr}
$\begin{array}{c}\text { Load } \\
\mathrm{g}\end{array}$ & $\begin{array}{c}\text { Protruding } \\
\mathrm{cm}^{3} \\
\times 10^{4}\end{array}$ & $\begin{array}{c}\text { Total } \\
\mathrm{g}\end{array}$ & $\begin{array}{c}\varrho_{20,20} \text { with } \\
\text { syst.error }\end{array}$ & $K \times 10^{5}$ & $\begin{array}{c}\varrho_{20,20} \text { corrected } \\
\text { for } \\
\text { syst.error }\end{array}$ & $\lambda \times 10^{5}$ \\
4.965 & 29 & 5.0679 & 0.99988 & 12 & 0.99998 & -2 \\
4.967 & 10 & 5.0680 & 0.99989 & 11 & 0.99999 & -1 \\
4.966 & 23 & 5.0683 & 0.99990 & 10 & 1.00000 & 0 \\
4.965 & 23 & 5.0673 & 0.99985 & 15 & 0.99995 & -5 \\
4.966 & 23 & 5.0683 & 0.99990 & 10 & 1.00000 & 0 \\
4.967 & 15 & 5.0685 & 0.99991 & 9 & 1.00001 & +1 \\
4.968 & 14 & 5.0694 & 0.99996 & 4 & 1.00006 & +6 \\
4.966 & 17 & 5.0677 & 0.99987 & 13 & 0.99997 & -3 \\
4.967 & 16 & 5.0686 & 0.99992 & 8 & 1.00002 & +2 \\
4.966 & 19 & 5.0679 & 0.99988 & 12 & 0.99998 & -2 \\
\hline \multicolumn{7}{r}{} \\
$\mu_{K, \mathrm{~m}}=10 \times 10^{-6}$ & 50.6819 & & 104 & & \\
$\mu_{\varrho_{\mathrm{f}, 20,20}=33 \times 10^{-6}}=3.06819$ & 0.999896 & 10 & &
\end{tabular}


Table 6. Hydrometer method, diver No. 37, diameter of stem $0.5 \mathrm{~mm}$, $\varrho_{20,20}=0.950156, C=20.0010$; weight of stem $0.500 \mathrm{~g}$.

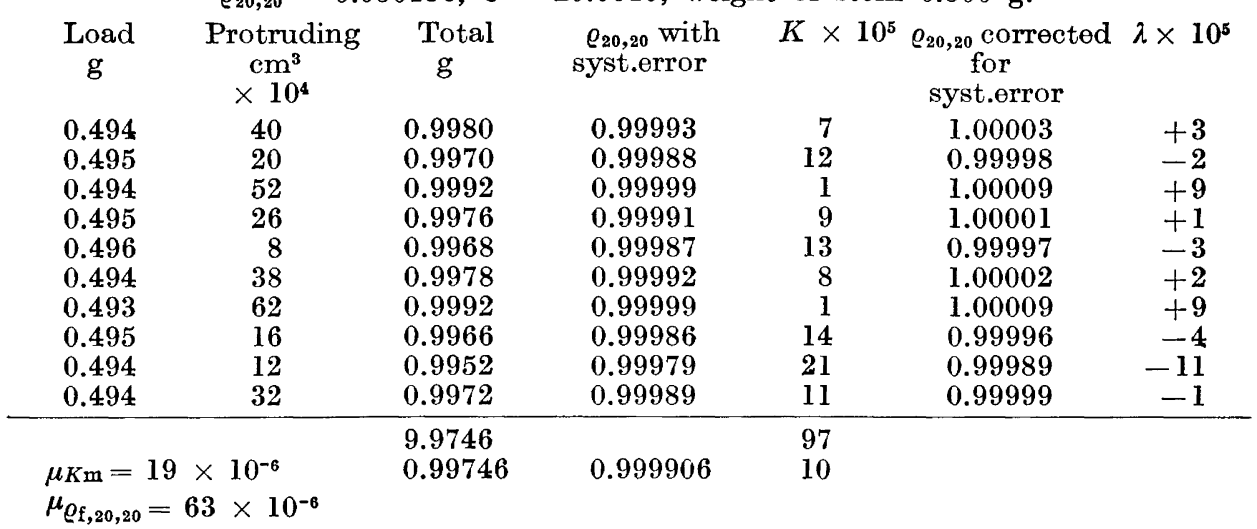

Table 7. Hydrometer method, diver No. 37, diameter of stem $0.3 \mathrm{~mm}$, $\varrho_{20,20}=0.950156, C=20.0010$; weight of stem $0.200 \mathrm{~g}$.

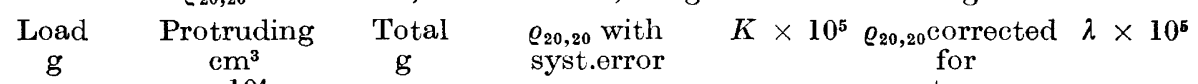
$\times 10^{4}$

\begin{tabular}{|c|c|c|c|}
\hline 0.796 & 22 & 0.9982 & 0.99994 \\
\hline 0.797 & 9 & 0.9979 & 0.99993 \\
\hline 0.798 & 3 & 0.9983 & 0.99995 \\
\hline 0.796 & 16 & 0.9976 & 0.99991 \\
\hline 0.795 & 19 & 0.9969 & 0.99988 \\
\hline 0.796 & 17 & 0.9977 & 0.99992 \\
\hline 0.797 & 11 & 0.9981 & 0.99994 \\
\hline 0.796 & 19 & 0.9979 & 0.99993 \\
\hline 0.795 & 22 & 0.9972 & 0.99989 \\
\hline 0.796 & 13 & 0.9973 & 0.99990 \\
\hline, $\mathbf{m}=$ & $10^{-6}$ & $\begin{array}{l}9.9771 \\
0.99771\end{array}$ & 0.999919 \\
\hline
\end{tabular}

$\mu_{Q_{\mathrm{f}, 20,20}}=25 \times 10^{-6}$

Table 8. Hydrometer method, diver No. 37, diameter of stem: $0.2 \mathrm{~mm}$, $\varrho_{20,20}=0.950156, C=20.0010$; weight of stem $0.100 \mathrm{~g}$.

Load Protruding Total $\varrho_{20,20}$ with $K \times 10^{5} \varrho_{20,20}$ corrected $\lambda \times 10^{5}$

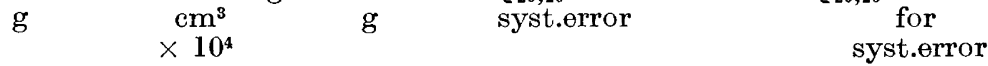

\begin{tabular}{|c|c|c|c|c|c|c|}
\hline 0.895 & 17 & 0.9967 & 0.99987 & 13 & 0.99998 & -2 \\
\hline 0.897 & 4 & 0.9974 & 0.99990 & 10 & 1.00001 & +1 \\
\hline 0.896 & 14 & 0.9974 & 0.99990 & 10 & 1.00001 & +1 \\
\hline 0.895 & 16 & 0.9966 & 0.99986 & 14 & 0.99997 & -3 \\
\hline 0.897 & 7 & 0.9977 & 0.99992 & 8 & 1.00003 & +3 \\
\hline 0.896 & 14 & 0.9974 & 0.99990 & 10 & 1.00001 & +1 \\
\hline 0.896 & 8 & 0.9968 & 0.99987 & 13 & 0.99998 & -2 \\
\hline 0.896 & 12 & 0.9972 & 0.99989 & 11 & 1.00000 & 0 \\
\hline 0.897 & 12 & 0.9982 & 0.99994 & 6 & 1.00005 & +5 \\
\hline 0.895 & 17 & 0.9967 & 0.99987 & 13 & 0.99998 & -2 \\
\hline$f, 20,20$ & $\begin{array}{l}10^{-6} \\
10^{-6}\end{array}$ & $\begin{array}{l}9.9721 \\
0.99721\end{array}$ & 0.999894 & $\begin{array}{r}108 \\
11\end{array}$ & & \\
\hline
\end{tabular}

Acta Chem. Scand. 12 (1958) No. 5 


$$
\mu \mathrm{K}_{\mathrm{m}}=\sqrt{\mu \mathrm{dm}^{2}+\frac{\sum_{1}^{\mathrm{n}} \lambda^{2}}{\mathrm{n}(\mathrm{n}-1)}}
$$

where $\mathrm{n}=10$ (and $\mu \mathrm{dm}$ is the random error of the determination of the specific gravity of the diver by 10 experiments).

Further the random error of a single determination $\mu_{\mathrm{f}, \mathbf{2 0}, 20}$ of the specific gravity $\varrho_{\mathrm{f}, 20,20}$ of water determined by the hydrometer method may be expressed by the equation

$$
\begin{aligned}
& \mu_{\varrho \mathrm{f}, 20,20}=\sqrt{\mu \mathrm{dm}^{2}+\mu_{K \mathrm{~m}^{2}}+\frac{\sum_{1{ }^{2} \lambda^{2}}^{\mathrm{n}}}{\mathrm{n}-\mathrm{l}}}= \\
& =\sqrt{2 \mu \mathrm{dm}^{2}+\frac{(\mathrm{n}+1) \sum_{1}^{\mathrm{n}}{ }^{2}}{\mathrm{n}(\mathrm{n}-1)}}
\end{aligned}
$$

where $n=10$.

The results of the experiments are given in Tables $3-8$.

The author gratefully acknowledges financial support from the Foundation for Applied Chemistry of the Technical University of Denmark.

\section{REFERENCES}

1. Marsigli, J. Histoire physique de la mer, Amsterdam 1725.

2. Pisati, G. and Reggiani N. Rend. accad. naz. Lincei 6 (1890) 99.

3. Nansen, F. The Norwegian North Polar Expedition, Scientific Results, Christiania and London 1902, Vol. III, p. 78.

4. Thoulet, J. Bull. Inst. Océanograph. (1910) No. 181.

5. Warrington, A. W. Phil. Mag. 48 (1899) 498.

6. Richards, T. W. and Shipley, J. W. J. Am. Chem. Soc. 34 (1912) 599.

7. Richards, T. W. and Shipley, J. W. J. Am. Chem. Soc. 36 (1914) 1.

8. Richards, T. W. and Harris, G. W. J. Am. Chem. Soc. 38 (1916) 1000.

9. Lamb, A. B. and Lөe, R. E. J. Am. Chem. Soc. 35 (1913) 1666.

10. Emeleus, H. J., James, F. W., King, A., Pearson, T. G., Purcell, R. H. and Briscoe, H. V. A. J. Chem. Soc. 19341207.

11. Anderson, J. S., Purcell, R. H., Pearson, T. G., King, A., James, F. W., Emeleus, H. J. and Briscoe, H. V. A. J. Chem. Soc. 19371492.

12. Berg, S. Studies on Particle-Size Distribution (Diss.) The Technical University of Denmark. G. E. C. Gad, Copenhagen 1940.

13. Berg, S. Untersuchungen über Korngrössenverteilung (Diss.) Kolloid-Beih. 53 (1941) 149.

14. Berg, S. Ber. deut. keram. Ges. 23 (1942) 271.

15. Berg, S. Transact. Intern. Ceram. Congr., Maestricht 1948.

16. Berg, S. Atti IV Congr. Intern. Ceram., Firenze 1954.

17. Berg, S. Ber. deut. keram. Ges. 33 (1956) 229.

Received February 2\%, 1958. 